\title{
Identification of genes associated with matrix metalloproteinases in invasive lung adenocarcinoma
}

\author{
WEIQING LI ${ }^{1}$, XUGANG ZHANG $^{1}$, ZHITIAN LI $^{1}$, FUSHENG JIANG $^{1}$, HONGWEI ZHAO $^{2}$ and BO WEI ${ }^{1}$ \\ Departments of ${ }^{1}$ Thoracic Surgery and ${ }^{2}$ Interventional Treatment, Beijing Shijitan \\ Hospital, Capital Medical University, Beijing 100038, P.R. China
}

Received August 17, 2015; Accepted February 7, 2017

DOI: $10.3892 / \mathrm{ol} .2018 .8683$

\begin{abstract}
The aim of the present study was to identify genes with similar function to that of matrix metalloproteinases (MMPs) in invasive lung adenocarcinoma (AC) and to screen the transcription factors that regulate MMPs. The gene expression dataset GSE2514, including 20 invasive lung AC samples and 19 adjacent normal lung samples, was downloaded from the Gene Expression Omnibus database. Differentially expressed genes (DEGs) were screened using the limma package in R. Genes with similar function to MMPs were identified by K-means clustering. Their correlations with MMPs were validated using Pearson correlation analysis. The expression of MMPs in lung cancer and normal tissues was evaluated by western blot analysis. Protein-protein interaction (PPI) network and transcriptional regulatory network analyses were performed with Retrieval of Interacting Genes and Database for Annotation, Visualization and Integrated Discovery, respectively. As a result, 269 DEGs were identified between invasive lung AC samples and normal lung samples, including 78 upregulated and 191 downregulated genes. Four MMPs (MMP1, MMP7, MMP9 and MMP12), which were upregulated in lung $\mathrm{AC}$, were clustered into one group with other genes, including $\mathrm{NAD}(\mathrm{P}) \mathrm{H}$ quinone oxidoreductase 1, claudin 3 (CLDN3), S100 calcium-binding protein P, serine protease inhibitor Kazal type 1, collagen type XI $\alpha 1$ chain, periostin and desmoplakin (DSP), following cluster analysis. Pearson correlation analysis further confirmed correlations between MMP9-CLDN3, MMP9-DSP and MMP12-DSP. PPI network analysis also indicated multiple interactions between MMPs-associated genes. Furthermore, MMPs were commonly regulated by CCAAT/enhancer binding protein $\alpha$ transcription
\end{abstract}

Correspondence to: Dr Bo Wei, Department of Thoracic Surgery, Beijing Shijitan Hospital, Capital Medical University, 10 Tieyi Road, Haidian, Beijing 100038, P.R. China

E-mail: boweibw@hotmail.com

Key words: lung adenocarcinoma, differentially expressed genes, matrix metalloproteinases, protein-protein interaction network, transcription factors factor. These findings may provide further insight into the mechanisms of MMPs in invasive lung AC.

\section{Introduction}

Lung cancer is a leading cause of cancer mortalities worldwide, with 226,160 new cases and 160,340 mortalities in 2012 (1). As a type of lung cancer, non-small cell lung carcinoma (NSCLC) consists of adenocarcinoma (AC), squamous cell carcinoma and large cell carcinoma (2). Lung AC, a heterogeneous group of tumors ranging in aggressiveness from noninvasive bronchioloalveolar carcinoma (BAC) to microinvasive tumors, mixed-type tumors and pure invasive $\mathrm{AC}$, is the most common histological type of lung cancer $(3,4)$. The first treatment for patients with early-stage lung $\mathrm{AC}$ is surgical resection, but the 5-year overall survival rate remains at $\sim 80 \%$ for stage IA disease (5). Notably, patients with invasive lung $\mathrm{AC}$ have a lower mean 5-year survival rate compared with that of patients with BAC (59 vs. 100\%, respectively) (6). Therefore, it is important to better understand the molecular mechanisms of invasive lung $\mathrm{AC}$ in order to develop effective preventive and curative strategies.

Cancer invasion is a highly complicated process, with the early events being the proteolytic degradation of extracellular matrix (ECM) components to provide room for infiltration, followed by migration into the adjacent tissues $(7,8)$. Recent studies suggest that matrix metalloproteinases (MMPs), members of the matrixin subfamily of zinc metalloproteinases, are involved in the breakdown of the $\operatorname{ECM}(9,10)$. Over-expression of MMPs has been detected in a number of tumor types, including invasive lung AC (11). MMP2, MMP7, MMP9 and tissue inhibitor of metalloproteinase 2 are upregulated in the lung tissue of patients with primary spontaneous pneumothorax (PSP), and the imbalance of their expression may be implicated in the pathogenesis of PSP (12). The signal module and sequence variant module appearing in lung $\mathrm{AC}$, in which the expression of MMP12 is upregulated but that of MMP11 is downregulated, can promote the invasion of cancer cells (13). Chemotherapy drugs can markedly inhibit the invasive ability of human lung AC cells via reducing the expression of MMP2 and MMP9 (14,15). Additionally, rosuvastatin and simvastatin can function in the treatment of lung cancer by regulating the expression of MMP2, MMP9, RAS and nuclear factor- $\kappa$ B-p65 (16). However, the current 
number of screened MMPs is limited, and further studies are required to identify genes with a similar function to that of MMPs.

In addition, it has been reported that the transcription factor signal transducer and activator of transcription 3 (STAT3) can regulate the transcription of MMP2 and promote melanoma cell invasion (17). Blocking STAT3 signaling significantly inhibits the invasion of melanoma cells (17). Storz et al (17) have demonstrated that forkhead box $\mathrm{O} 3$ promotes invasion and progression in numerous human solid tumors by inducing the expression of MMP9 and MMP13. However, the regulatory mechanisms of MMPs are not well understood in lung AC.

The present study aimed to identify differentially expressed genes (DEGs), particularly MMPs-associated genes, using gene expression profile data of invasive lung AC and adjacent normal samples collected from a publicly available database. Besides, the transcriptional factors regulating MMPs-associated genes were also investigated.

\section{Materials and methods}

Ethical statement. The present study was approved by the Ethics Committees of the Beijing Shijitan Hospital (Beijing, China) and the Chest Hospital Affiliated to Shanghai Jiaotong University (Shanghai, China), and it was performed in accordance with the ethical standards (18). In addition, written informed consent was obtained from all patients, prior to enrollment in the present study.

Microarray data. The gene expression dataset GSE2514, which is based on two platforms [GPL81 (MG_U74Av2) Affymetrix Murine Genome U74A Version 2 Array and GPL8300 (HG_U95Av2) Affymetrix Human Genome U95 Version 2 Array], was downloaded from Gene Expression Omnibus (GEO; https://www.ncbi.nlm.nih.gov/geo/) database (19). Microarray data obtained with the platform GPL8300 (HG U95Av2; Affymetrix Human Genome U95 Version 2 Array; Affymetrix, Inc., Santa Clara, CA, USA) was downloaded from the GEO database (19) in our study. It contained 20 lung AC samples and 19 adjacent lung samples $(\sim 1 \mathrm{~cm}$ away from the tumor site), which were obtained from 5 male and 5 female patients undergoing lobectomy (9) and wedge resection (1). Of these patients, 9 had a history of tobacco smoking. The ages of the patients ranged from 45 to 73 years. Their tumors were all invasive lung $\mathrm{AC}$. The majority of tumors were low-to-intermediate grade and low stage, although 2 stage III tumors were included in the analysis. Probe annotation files were also acquired.

Preprocessing and differential analysis. The raw array data in the CEL file (Affymetrix, Inc.) were transformed into recognizable gene expression data using the robust multi-array average algorithm from the affy package in R (20). Upon normalization, probes were mapped to genes according to the annotation files. The levels of probes corresponding to one gene were averaged and used as the final gene expression value. DEGs were screened using the limma package (http://www.bioconductor.org/packages/release/bioc/html/limma.html) (21) in R based on the cut-offs of $\mathrm{P}<0.05$ and log fold-changel $>1$.
Cluster analysis. Cluster analysis of DEGs was performed with Cluster 3.0 (http://bonsai.ims.u-tokyo.ac.jp/mdehoon/software/cluster) using the K-means clustering algorithm (22), which was conducted on data with $\mathrm{K}$ (the number of clusters) $=5$.

Pearson correlation analysis. The method of Pearson correlation analysis (23) in the cor function in R (https://www.r-project .org/) was used to perform the correlation analysis between the expression of DEGs and MMPs. A correlation coefficient of $>0.8$ was used as the cut-off criterion.

Western blot analysis. A total of 6 lung AC and matched adjacent lung tissue biopsy samples were obtained in June 2014 from Shanghai Lung Cancer Center, Chest Hospital Affiliated to Shanghai Jiaotong University (Shanghai, China). Tissues were washed with ice-cold PBS and lysed in ice-cold radioimmunoprecipitation assay lysis buffer (Sangon Biotech Co., Ltd., Shanghai, China). Extracted proteins were quantified using a BCA Protein Assay kit (Sangon Biotech Co., Ltd., Shanghai, China) and separated with SDS-PAGE with a $10 \%$ separating gel and a 5\% stacking gel. Subsequently, proteins were transferred onto polyvinylidene difluoride membranes (EMD Millipore, Billerica, MA, USA). Then, membranes were blocked in 5\% nonfat dry milk and probed with primary rabbit antibodies against MMPs (anti-MMP1, cat. no. sc-58377; anti-MMP7, cat. no. sc-80205; anti-MMP9, cat. no. sc-13520; and anti-MMP12, cat. no. sc-8839; all diluted to 1:500; Santa Cruz Biotechnology, Inc., Dallas, TX, USA) overnight at $4^{\circ} \mathrm{C}$. Horseradish peroxidase-conjugated anti-rabbit immunoglobulin $\mathrm{G}$ (cat. no. 111-035-003; 1:5,000; Jackson ImmunoResearch Laboratories, Inc., West Grove, PA, USA) served as a secondary antibody and was incubated for $1 \mathrm{~h}$ at $4^{\circ} \mathrm{C}$. The anti- $\beta$-actin antibody (cat. no. 8227; 1:10,000; Abcam, Cambridge, CA, USA) was used as the control. The position of protein bands was developed with ECL chemoluminescence kit (Merck Millipore) and visualized under a ChemiDoc MP imaging system (Bio-Rad Laboratories, Inc., Hercules, CA, USA).

Functional and pathway enrichment analysis. Gene Ontology (GO; http://www.geneontology.org/) (24) and Kyoto Encyclopedia of Genes and Genomes (KEGG; http://www .genome.jp/kegg/) (25) pathway enrichment analyses were performed for the MMPs-associated genes using the Database for Annotation, Visualization and Integrated Discovery (DAVID) online tool (26) to reveal altered biological functions in lung AC. $\mathrm{P}<0.05$ and a false discovery rate (FDR) adjusted by the Benjamini and Hochberg method (27) of $<0.01$ were set as the thresholds.

Selection of disease-associated genes. Diseases associated with the DEGs were identified by gene set enrichment analysis against the Genetic Association Database Disease Class (http://geneticassociationdb.nih.gov/) using the annotation server DAVID $(\mathrm{P}<0.05)(26)$.

Construction of protein-protein interaction (PPI) network. PPI network analysis for the DEGs was carried out with the Search Tool for the Retrieval of Interacting Genes (STRING; http://string-db.org/), and the PPI network involving 


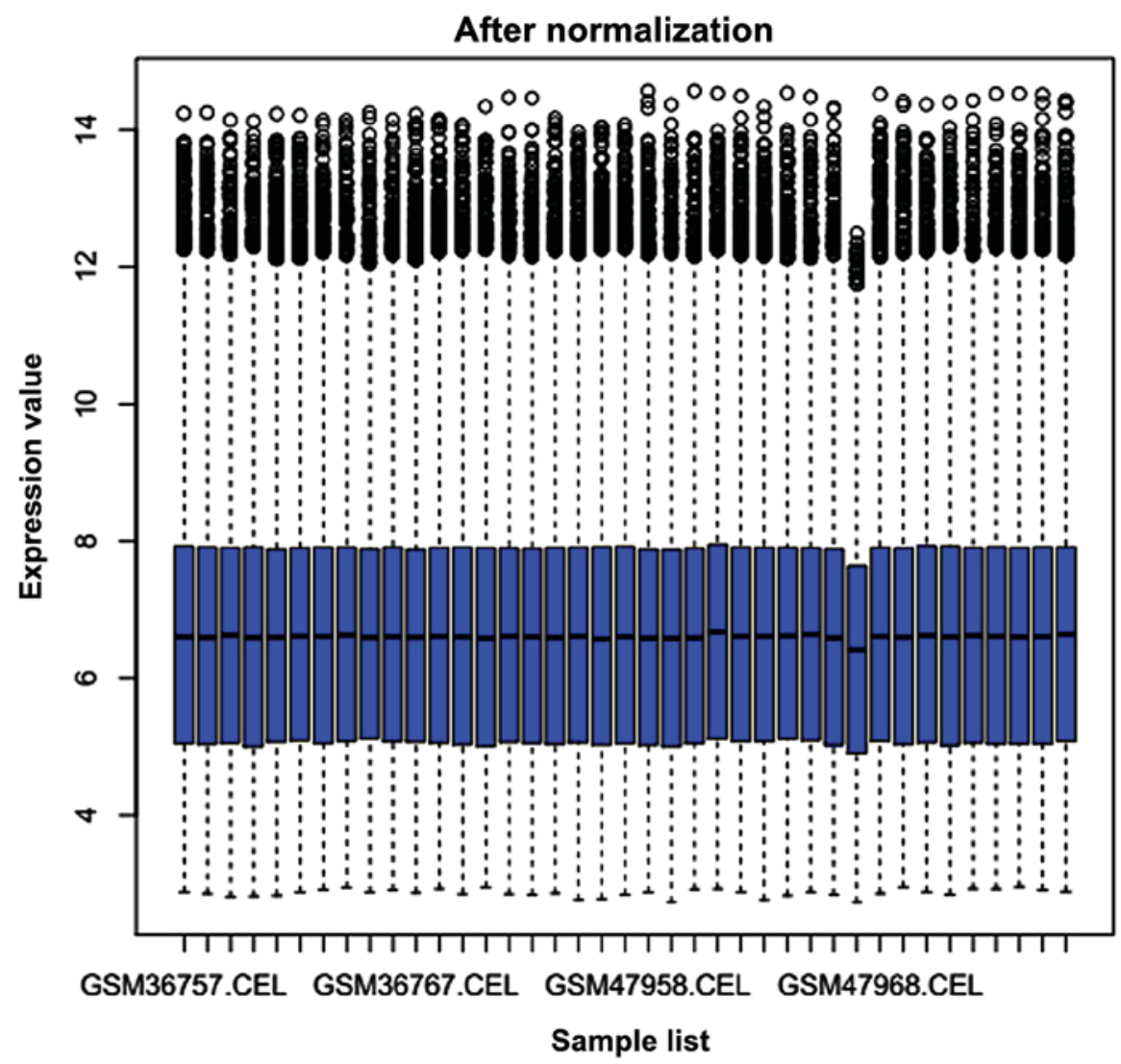

Figure 1. Box plot for normalized gene expression data. Horizontal axis and vertical axis separately represent sample names and expression values. The blue box and the black line represent expression values and their median, respectively. The medians (black lines) are almost all at the same level, indicating a good performance of normalization.

MMPs-associated genes was selected. Interaction associations with a combined score of $\geq 0.4$ were retained and the PPI network was visualized by Cytoscape software (version 2.8; http://www.cytoscape.org) (28).

Transcriptional regulatory network analysis. Transcriptional regulatory network analysis was performed with DAVID (26) for the group of DEGs including MMPs, and the transcriptional regulatory network was visualized by Cytoscape software (version 2.8; http://www.cytoscape.org) (28).

\section{Results}

DEGs analysis. Upon normalization of the raw data (Fig. 1), the DEGs were screened using the limma package in R. As a result, 269 DEGs were identified between lung AC and adjacent normal lung samples, including 78 upregulated and 191 downregulated genes.

MMPs-associated genes screening. MMP1, MMP7, MMP9 and MMP12 were clustered into one group with a number of other DEGs (Table I), which were upregulated in lung AC compared with their expression in normal lung tissues. These results were further confirmed by Pearson correlation analysis and western blotting. The present findings also demonstrated that MMP9 expression exhibited a significant correlation with DSP (correlation coefficient $=0.8309295$ ) and CLDN3 (correlation coefficient $=0.8058015$ ) expression. Additionally, MMP12 expression was significantly correlated with DSP expression (correlation coefficient=0.8127249). The expression of MMP1, MMP7, MMP9 and MMP12 was also observed to be upregulated in lung $\mathrm{AC}$ compared with that in adjacent lung tissues (Fig. 2).

Functional and pathway enrichment analysis. Functional enrichment analysis was applied for the above group of DEGs with the DAVID online tool. As shown in Table II, collagen metabolism $\left(\mathrm{P}=4.37 \times 10^{-6}\right)$ and multicellular organism macromolecule metabolic processes $\left(\mathrm{P}=5.98 \times 10^{-6}\right)$ were enriched for these DEGs.

Disease-relevant genes selection. Relevant diseases to the MMPs-associated DEGs were retrieved with DAVID. Under the Genetic Association DB Disease Class, MMP9, NAD(P)H quinone oxidoreductase 1 (NQO1), MMP12 and MMP1 were linked with lung AC, while MMP9, MMP12 and MMP1 were associated with lung function (Table III).

PPI network analysis. A PPI network was constructed for the MMPs-associated DEGs using STRING (Fig. 3). The results revealed that interaction associations existed among MMP9, NQO1 and MMP7. In addition, the other genes clustered into the MMPs group also interacted with each other.

Transcriptional regulatory network analysis. Transcriptional regulatory network analysis indicated that 9 genes, including 
Table I. List of genes clustered with MMPs.

\begin{tabular}{|c|c|c|c|c|c|}
\hline ID & Log (fold-change) & Mean expression & $\mathrm{t}$ & P-value & False discovery rate \\
\hline RAMP1 & 1.803939312 & 6.214583105 & -10.555338710 & $4.33 \times 10^{-13}$ & $3.50 \times 10^{-11}$ \\
\hline FAP & 1.561195027 & 6.500446054 & -9.026036586 & $3.63 \times 10^{-11}$ & $1.48 \times 10^{-9}$ \\
\hline CLDN3 & 1.625063261 & 9.253029938 & -7.959600860 & $9.43 \times 10^{-10}$ & $2.41 \times 10^{-8}$ \\
\hline $\mathrm{CP}$ & 1.533166764 & 7.200364641 & -7.654471654 & $2.45 \times 10^{-9}$ & $5.46 \times 10^{-8}$ \\
\hline ZWINT & 1.534376695 & 6.188206312 & -7.484374926 & $4.18 \times 10^{-9}$ & $8.53 \times 10^{-8}$ \\
\hline MDK & 1.627179122 & 9.344798091 & -7.324035903 & $6.94 \times 10^{-9}$ & $1.32 \times 10^{-7}$ \\
\hline MMP9 & 1.708966359 & 7.597559232 & -6.771606537 & $4.04 \times 10^{-8}$ & $5.82 \times 10^{-7}$ \\
\hline COL3A1 & 1.643158431 & 9.138801925 & -6.532240647 & $8.72 \times 10^{-8}$ & $1.11 \times 10^{-6}$ \\
\hline COL11A1 & 2.387669841 & 5.767529739 & -6.412529728 & $1.28 \times 10^{-7}$ & $1.54 \times 10^{-6}$ \\
\hline TOX3 & 1.770307511 & 4.159327973 & -6.212832065 & $2.44 \times 10^{-7}$ & $2.68 \times 10^{-6}$ \\
\hline GABBR1 UBD & 1.619335627 & 7.242024866 & -6.016224500 & $4.60 \times 10^{-7}$ & $4.64 \times 10^{-6}$ \\
\hline MMP12 & 1.644563956 & 5.138055675 & -5.757801490 & $1.06 \times 10^{-6}$ & $9.61 \times 10^{-6}$ \\
\hline POSTN & 1.502940090 & 7.019690170 & -5.698113750 & $1.28 \times 10^{-6}$ & $1.13 \times 10^{-5}$ \\
\hline SPINK1 & 2.517220534 & 7.945875667 & -5.613361864 & $1.69 \times 10^{-6}$ & $1.44 \times 10^{-5}$ \\
\hline NQO1 & 1.527576974 & 7.820504924 & -5.374097053 & $3.64 \times 10^{-6}$ & $2.77 \times 10^{-5}$ \\
\hline SLC7A5 & 1.517121297 & 7.685388227 & -5.301930776 & $4.59 \times 10^{-6}$ & $3.37 \times 10^{-5}$ \\
\hline MMP7 & 2.087445737 & 6.987333841 & -5.300686125 & $4.61 \times 10^{-6}$ & $3.38 \times 10^{-5}$ \\
\hline MMP1 & 1.553980336 & 4.289661258 & -5.235072252 & $5.68 \times 10^{-6}$ & $4.05 \times 10^{-5}$ \\
\hline DSP & 1.669305242 & 7.306405073 & -5.162024033 & $7.18 \times 10^{-6}$ & $4.94 \times 10^{-5}$ \\
\hline CEACAM5 & 2.090196480 & 7.403316921 & -4.560737891 & $4.80 \times 10^{-5}$ & $2.43 \times 10^{-4}$ \\
\hline S100P & 1.513031206 & 7.826887416 & -3.965427875 & $2.97 \times 10^{-4}$ & $1.12 \times 10^{-3}$ \\
\hline
\end{tabular}

$\mathrm{t}$, (the sample mean-the expected value for the population mean)/the standard error of the mean. MMP, matrix metalloproteinase; ID, identity; RAMP1, receptor activity modifying protein 1; FAP, familial adenomatous polyposis; CLND3, claudin 3; CP, ceruloplasmin; ZWINT, ZW10 interacting kinetochore protein; MDK, midkine; COL3A1, collagen type III $\alpha$ 1; COL11A1, collagen type XI $\alpha$ I; TOX3, TOX high mobility group box family member 3; UBD, ubiquitin D; POSTN, periostin; SPINK1, serine peptidase inhibitor, Kazal type 1; NQO1, NAD(P)H quinone oxidoreductase 1; SLC7A5, solute carrier family 7 member 5; DSP, desmoplakin; CEACAM5, carcinoembryonic antigen-related cell adhesion molecule 5; S100P, S100 calcium-binding protein P.

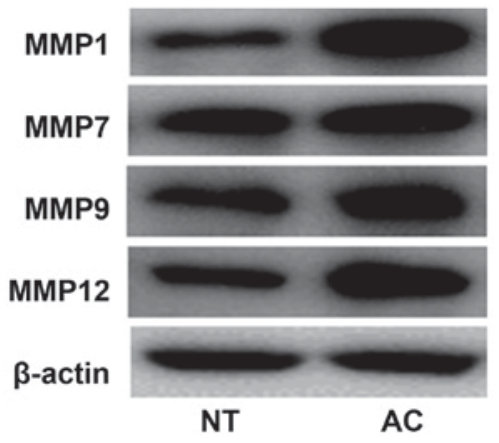

Figure 2. Expression of MMPs (MMP1, MMP7, MMP9 and MMP12) in lung AC tissues and adjacent NTs evaluated by western blotting. AC, adenocarcinoma; NT, normal tissue; MMP, matrix metalloproteinase.

MMP1, MMP9 and NQO1, were commonly regulated by the CCAAT/enhancer binding protein (C/EBP) $\alpha$ (CEBPA) transcription factor (Fig. 4).

\section{Discussion}

In the present study, cluster analysis demonstrated that 4 MMPs, including MMP1, MMP7, MMP9 and MMP12, were upregulated in lung AC samples, and may be involved in ECM metabolic processes, thus promoting cancer invasion. These results were validated by western blotting and were in accordance with previous studies (29-32). MMP1 is the most highly expressed interstitial collagenase for degrading fibrillar collagens (33). Overexpression of MMP1 has been associated with tumor invasion and metastasis by modulating the polarization of $\mathrm{T}$ helper $(\mathrm{Th}) 1 / \mathrm{Th} 2$ inflammatory responses (29). Downregulation of MMP-7 mediated by antisense oligonucleotide changes the ultrastructure of lung AC A549 cells, leading to decreased microvilli, endoplasmic reticulum dilation, swelling of mitochondria and formation of apoptotic bodies, which eventually inhibits invasion in lung AC A549 cells (30). Similarly, the expression levels of MMP9 and MMP12 were observed to be higher in NSCLC than in normal samples (31). Upregulation of MMP12 and MMP9 may be one of the mechanisms to promote lung cancer cell invasion (32).

In addition, the present study identified several DEGs that were clustered into one group with MMPs, thus indicating the same function in cancer cell invasion. A number of these genes have been linked to lung cancer in the following studies. NQO1 is a member of the NQO family, and altered expression of this protein has been reported in numerous tumors (34). Mutations 
Table II. GO terms over-represented for the MMPs-associated genes.

\begin{tabular}{|c|c|c|c|c|c|c|}
\hline \multirow[b]{2}{*}{ Category } & \multirow[b]{2}{*}{ Term } & \multicolumn{2}{|c|}{ Gene } & \multirow[b]{2}{*}{ P-value } & \multirow[b]{2}{*}{ Genes } & \multirow[b]{2}{*}{ False discovery rate } \\
\hline & & $\mathrm{N}$ & $\%$ & & & \\
\hline GO_BP & $\begin{array}{l}\text { GO:0032963 collagen } \\
\text { metabolic process }\end{array}$ & 4 & 20 & $4.37 \times 10^{-6}$ & $\begin{array}{l}\text { MMP9, COL } 3 \mathrm{~A} 1 \text {, } \\
\text { MMP7 and MMP1 }\end{array}$ & 0.005547327 \\
\hline GO_BP & $\begin{array}{l}\text { GO:0044259 multicellular } \\
\text { organism macromolecule } \\
\text { metabolic process }\end{array}$ & 4 & 20 & $5.98 \times 10^{-6}$ & $\begin{array}{l}\text { MMP9, COL3A1, } \\
\text { MMP7 and MMP1 }\end{array}$ & 0.007594962 \\
\hline GO_CC & $\begin{array}{l}\text { GO: } 0005578 \text { proteinaceous } \\
\text { ECM }\end{array}$ & 7 & 35 & $2.30 \times 10^{-6}$ & $\begin{array}{l}\text { MMP9, COL } 3 \mathrm{~A} 1 \text {, } \\
\text { MMP7, POSTN, COL11A1, } \\
\text { MMP12 and MMP1 }\end{array}$ & 0.002198433 \\
\hline GO_CC & GO:0031012 ECM & 7 & 35 & $3.56 \times 10^{-6}$ & $\begin{array}{l}\text { MMP9, COL } 3 \mathrm{~A} 1 \text {, } \\
\text { MMP7, POSTN, COL11A1, } \\
\text { MMP12 and MMP1 }\end{array}$ & 0.003399869 \\
\hline
\end{tabular}

Go, Gene Ontology; BP, biological process; CC, cellular component; ECM, extracellular matrix; MMP, matrix metalloproteinase; COL3A1; collagen, type III $\alpha 1$; POSTN, periostin; COL11A1, collagen type XI $\alpha$ I.
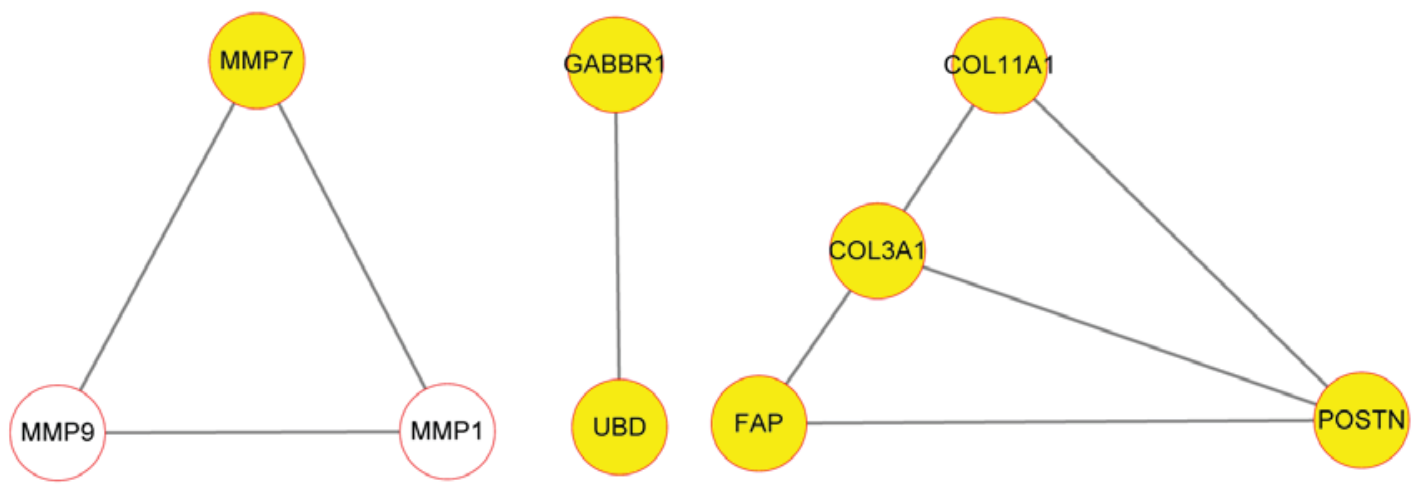

Figure 3. Protein-protein interaction network for MMPs-associated genes. MPPs are marked in circles and other genes in squares. Lung cancer-associated genes are presented as white and other genes as yellow. MMP, matrix metalloproteinase; GABBR1, $\gamma$-aminobutyric acid type B receptor subunit 1 ; UBD, ubiquitin D; FAP, familial adenomatous polyposis; COL11A1, collagen type XI $\alpha$ I; COL3A1, collagen type III $\alpha$; POSTN, periostin.

in this gene contribute to susceptibility to various forms of cancer, including lung cancer $(35,36)$. S100 calcium-binding protein $\mathrm{P}(\mathrm{S} 100 \mathrm{P})$ is a member of the $\mathrm{S} 100$ family of proteins containing two EF-hand calcium-binding motifs, which are involved in the regulation of cell cycle progression and differentiation (37). Bartling et al (31) reported that S100P expression is mainly increased in $\mathrm{AC}$, and that S100P upregulation is detected in early rather than in advanced tumor stages. Overexpression of S100P may lead to cancer cell invasion by changing the expression levels of several cytoskeletal proteins (38). Serine peptidase inhibitor, Kazal type 1 (SPINK1) is a secreted serine protease inhibitor (39). Overexpression of SPINK1 is associated with aggressiveness of prostate cancer (40). A previous study by Soon et al (34) revealed that SPINK1 is an invasion factor associated with prognosis in breast cancer patients. However, its role in lung cancer remains unknown. The present study suggested that SPINK1 may also be involved in lung AC invasion. Ubiquitin D (UBD, also known as F-associated transcript 10) is a member of the ubiquitin-like modifier family, and appears to be upregulated in hepatocellular carcinoma, as well as in gastrointestinal and gynecological cancers (41). Increased cytoplasmic UBD is significantly associated with depth of colon cancer invasion (42). Few studies have investigated the roles of UBD in lung AC invasion, including the present study. Collagen type XI $\alpha$ I (COL11A1) has also been demonstrated to be overexpressed in NSCLC, and has been identified as a potential invasion-associated gene in cancer (43). However, its mechanism of function is not understood yet. The present study revealed that COL11A1 could interact with the osteoblast-specific factor periostin (POSTN), although this requires further experimental validation. In addition, the associations between MMPs and various genes (including MMP9-CLDN3, MMP9-DSP and MMP12-DSP) were also further confirmed by Pearson correlation analysis. CLDN3, a component of tight junctions, has been reported to be upregulated in NSCLC, and may be important in invasion (44). Agarwal et al (39) reported that CLDN3-mediated increased invasion may be accomplished through the activation of MMP2. In the present study, CLDN3 was significantly correlated with MMP9 
Table III. Diseases associated with MMPs-associated genes.

\begin{tabular}{|c|c|c|}
\hline Term & P-value & Genes \\
\hline Nasopharyngeal cancer & $8.72 \times 10^{-6}$ & MMP9, MMP7, NQO1, MMP12 and MMP1 \\
\hline Abdominal aortic aneurysm & $1.18 \times 10^{-4}$ & MMP9, COL3A1, MMP12 and MMP1 \\
\hline Brain cancer & $6.15 \times 10^{-4}$ & MMP9, MMP7, NQO1 and MMP1 \\
\hline Gastric ulcer & $6.37 \times 10^{-4}$ & MMP9, MMP7 and MMP1 \\
\hline Subarachnoid hemorrhage & $1.68 \times 10^{-3}$ & MMP9, MMP12 and MMP1 \\
\hline Lung function & $3.48 \times 10^{-3}$ & MMP9, MMP12 and MMP1 \\
\hline Ovarian cancer & $3.78 \times 10^{-3}$ & MMP9, MMP7, NQO1 and MMP1 \\
\hline Bladder cancer & $5.85 \times 10^{-3}$ & MMP9, NQO1, MMP12 and MMP1 \\
\hline Colorectal cancer & $6.11 \times 10^{-3}$ & MMP9, MMP7, NQO1, MDK and MMP1 \\
\hline Coronary artery luminal dimensions & $7.91 \times 10^{-3}$ & MMP7 and MMP12 \\
\hline Osseointegrated implant failure & $7.91 \times 10^{-3}$ & MMP9 and MMP1 \\
\hline Alzheimer's disease dementia, vascular & $1.18 \times 10^{-2}$ & MMP9 and MMP1 \\
\hline Chronic obstructive pulmonary disease & $1.84 \times 10^{-2}$ & MMP9, MMP12 and MMP1 \\
\hline Rheumatoid arthritis & $1.85 \times 10^{-2}$ & MMP9, MMP7, MMP12 and MMP1 \\
\hline Uterine leiomyoma & $1.97 \times 10^{-2}$ & MMP9 and MMP1 \\
\hline Adenomyosis endometriosis & $1.97 \times 10^{-2}$ & MMP9 and MMP7 \\
\hline Breast cancer & $2.09 \times 10^{-2}$ & MMP9, POSTN, NQO1, MMP12 and MMP1 \\
\hline Cervical artery dissection, spontaneous & $2.36 \times 10^{-2}$ & MMP9 and COL3A1 \\
\hline Aneurysm & $2.36 \times 10^{-2}$ & MMP9 and MMP12 \\
\hline Lung cancer & $2.51 \times 10^{-2}$ & MMP9, NQO1, MMP12 and MMP1 \\
\hline Coronary artery disease & $3.07 \times 10^{-2}$ & MMP9, COL3A1 and MMP12 \\
\hline H. pylori infection stomach cancer & $3.52 \times 10^{-2}$ & MMP9 and MMP7 \\
\hline Left ventricular remodeling & $3.90 \times 10^{-2}$ & MMP9 and MMP1 \\
\hline
\end{tabular}

MMP, matrix metalloproteinase; MDK, midkine; COL3A1, collagen type III $\alpha$ 1; POSTN, periostin; NQO1, NAD(P)H quinone oxidoreductase 1 .

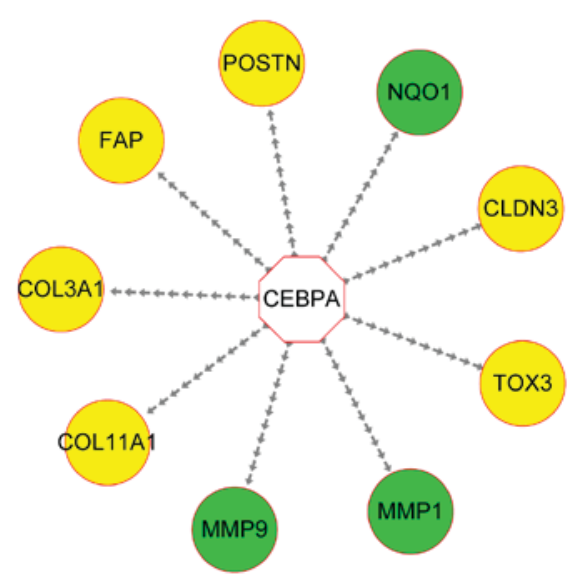

Figure 4. Transcriptional regulatory network containing MMPs. The transcription factor CEBPA is marked as white, while lung cancer-associated genes are presented as green and other genes as yellow. The dashed arrows indicate regulatory associations. MMP, matrix metalloproteinase; FAP, familial adenomatous polyposis; NQO1, NAD $(\mathrm{P}) \mathrm{H}$ quinone oxidoreductase 1; POSTN, periostin; COL11A1, collagen type XI $\alpha$ I; COL3A1, collagen type III $\alpha$ 1; TOX3, TOX high mobility group box family member 3; CLDN3, claudin 3; CEBPA, CCAAT/enhancer binding protein (C/EBP) $\alpha$.

via Pearson correlation analysis, indicating a potential link between CLDN3 and MMP9 in lung AC cell invasion. DSP acts as a tumor suppressor via inhibiting the Wnt/ $\beta$-catenin signaling pathway in NSCLC (45). Thus, the expression patterns of DSP and MMPs are theoretically opposite, which has been demonstrated in the human epithelial carcinoma cell line A431 during the epithelial-mesenchymal transition, with upregulated MMPs and downregulated DSP levels (46).

According to the present transcriptional regulatory network analysis, MMPs-associated genes were regulated by CEBPA. CEBPA is a basic leucine zipper domain transcription factor that not only can bind to certain promoters and enhancers as a homodimer, but can also form heterodimers with the associated proteins CEBPB and CEBPG (47). It has been reported that CEBPB is an important mediator in the activation of MMP genes (including MMP1, MMP3 and MMP10) in A549 lung carcinoma cells stimulated with the inflammatory cytokine interleukin-1 $\beta$ (48). Therefore, it could be speculated that CEBPA may also serve an important role in upregulating the expression of MMP1 and MPP9, thus affecting the invasion of lung cancer. Although the present results indicated that CEBPA could modulate the expression of fibroblast activation protein $\alpha$, CLDN3, collagen type III $\alpha 1$, COL11A1, TOX high mobility group box family member 3, NQO1 and POSTN, no experimental evidence could be obtained.

In conclusion, the present study identified several genes such as SPINK1 and UBD that may serve important roles in lung AC invasion with MMPs (including MMP1, MMP7, MMP9 and MMP12). Several MMPs-associated genes were observed 
to regulated by the CEBPA transcription factor. These findings may provide various underlying targets for prevention of lung $\mathrm{AC}$ invasion. However, further experimental investigations or studies on other datasets are required to validate the present observations.

\section{References}

1. Siegel R, Naishadham D and Jemal A: Cancer statistics, 2012. CA Cancer J Clin 62: 10-29, 2012.

2. Lazar V, Suo C, Orear C, van den Oord J, Balogh Z, Guegan J, Job B, Meurice G, Ripoche H, Calza S, et al: Integrated molecular portrait of non-small cell lung cancers. BMC Med Genomics 6 : 53, 2013.

3. Devesa SS, Bray F, Vizcaino AP and Parkin DM: International lung cancer trends by histologic type: Male:female differences diminishing and adenocarcinoma rates rising. Int J Cancer 117: 294-299, 2005

4. Borczuk AC, Qian F, Kazeros A, Eleazar J, Assaad A, Sonett JR, Ginsburg M, Gorenstein L and Powell CA: Invasive size is an independent predictor of survival in pulmonary adenocarcinoma. Am J Surg Pathol 33: 462-469, 2009.

5. van Rens MT, de la Rivière $A B$, Elbers HR and van Den Bosch JM: Prognostic assessment of 2,361 patients who underwent pulmonary resection for non-small cell lung cancer, stage I, II and IIIA. Chest 117: 374-379, 2000.

6. Sakurai H, Maeshima A, Watanabe S, Suzuki K, Tsuchiya R, Maeshima AM, Matsuno Y and Asamura H: Grade of stromal invasion in small adenocarcinoma of the lung: Histopathological minimal invasion and prognosis. Am J Surg Pathol 28: 198-206, 2004.

7. Veiseh O, Kievit FM, Ellenbogen RG and Zhang M: Cancer cell invasion: Treatment and monitoring opportunities in nanomedicine. Adv Drug Deliv Rev 63: 582-596, 2011

8. Kam Y, Rejniak KA and Anderson AR: Cellular modeling of cancer invasion: Integration of in silico and in vitro approaches. J Cell Physiol 227: 431-438, 2012.

9. Freije JM, Balbin M, Pendás AM, Sánchez LM, Puente XS and López-Otin C: Matrix metalloproteinases and tumor progression. Adv Exp Med Biol 532: 91-107, 2003.

10. Kleiner DE and Stetler-Stevenson WG: Matrix metalloproteinases and metastasis. Cancer Chemother Pharmacol 43 (Suppl): S42-S51, 1999.

11. Kumaki F, Matsui K, Kawai T, Ozeki Y, Yu ZX, Ferrans VJ and Travis WD: Expression of matrix metalloproteinases in invasive pulmonary adenocarcinoma with bronchioloalveolar component and atypical adenomatous hyperplasia. Am J Pathol 159: 2125-2135, 2001

12. Chen CK, Chen PR, Huang HC, Lin YS and Fang HY: Overexpression of matrix metalloproteinases in lung tissue of patients with primary spontaneous pneumothorax. Respiration 88: 418-425, 2014.

13. Sun Y, Wang L, Jiang M, Huang J, Liu Z and Wolfl S: Secreted phosphoprotein 1 upstream invasive network construction and analysis of lung adenocarcinoma compared with human normal adjacent tissues by integrative biocomputation. Cell Biochem Biophys 56: 59-71, 2010.

14. Yan KH, Lee LM, Yan SH, Huang HC, Li CC, Lin HT and Chen PS: Tomatidine inhibits invasion of human lung adenocarcinoma cell A549 by reducing matrix metalloproteinases expression. Chem Biol Interact 203: 580-587, 2013.

15. Kao SJ, Su JL, Chen CK, Yu MC, Bai KJ, Chang JH, Bien MY, Yang SF and Chien MH: Osthole inhibits the invasive ability of human lung adenocarcinoma cells via suppression of NF- $\mathrm{BB}$-mediated matrix metalloproteinase-9 expression. Toxicol Appl Pharmacol 261: 105-115, 2012.

16. Falcone D, Gallelli L, Di Virgilio A, Tucci L, Scaramuzzino M, Terracciano R, Pelaia G and Savino R: Effects of simvastatin and rosuvastatin on RAS protein, matrix metalloproteinases and $\mathrm{NF}-\kappa \mathrm{B}$ in lung cancer and in normal pulmonary tissues. Cell Prolif 46: 172-182, 2013.

17. Storz P, Döppler H, Copland JA, Simpson KJ and Toker A: FOXO3a promotes tumor cell invasion through the induction of matrix metalloproteinases. Mol Cell Biol 29: 4906-4917, 2009.

18. Assembly WMAG: World medical association declaration of Helsinki: Ethical principles for medical research involving human subjects. Chinese J Integrative Med 310: 2191-2194, 2001 .
19. Stearman RS, Dwyer-Nield L, Zerbe L, Blaine SA, Chan Z, BunnPAJr,Johnson GL, HirschFR, Merrick DT, Franklin WA, et al: Analysis of orthologous gene expression between human pulmonary adenocarcinoma and a carcinogen-induced murine model. Am J Pathol 167: 1763-1775, 2005.

20. Gautier L, Cope L, Bolstad BM and Irizarry RA: Affy-analysis of Affymetrix GeneChip data at the probe level. Bioinformatics 20: 307-315, 2004.

21. Ritchie ME, Phipson B, Wu D, Hu Y, Law CW, Shi W and Smyth GK: limma powers differential expression analyses for RNA-sequencing and microarray studies. Nucleic Acids Res 43: e47, 2015 .

22. Na S, Xumin L and Yong G: Research on k-means clustering algorithm: An improved k-means clustering algorithm. In: Proceedings of the Third International Symposium on Intelligent Information Technology and Security Informatics (IITSI). IITSI, Jian, pp63S-67S, 2010.

23. Månsson R, Tsapogas P, Akerlund M, Lagergren A, Gisler R and Sigvardsson M: Pearson correlation analysis of microarray data allows for the identification of genetic targets for early B-cell factor. J Biol Chem 279: 17905-17913, 2004.

24. Sherlock G: Gene Ontology: Tool for the unification of biology. Canadian Institute Food Sci Technol J 22: 415, 2009.

25. Kanehisa M, Sato Y, Kawashima M, Furumichi M and Tanabe M: KEGG as a reference resource for gene and protein annotation. Nucleic Acids Res 44: D457-D462, 2016.

26. Huang da W, Sherman BT and Lempicki RA: Systematic and integrative analysis of large gene lists using DAVID bioinformatics resources. Nature Protoc 4: 44-57, 2009.

27. Benjamini $\mathrm{Y}$ and Hochberg Y: Controlling the false discovery rate: A practical and powerful approach to multiple testing. J Royal Statistical Society 57: 289-300, 1995

28. Yeung N, Cline MS, Kuchinsky A, Smoot ME and Bader GD Exploring biological networks with Cytoscape software. Curr Protoc Bioinformatics 23: Chapter 8: 8.13:8.13.1-8.13.20, 2008.

29. Fanjul-Fernández M, Folgueras AR, Fueyo A, Balbín M, Suárez MF, Fernández-García MS, Shapiro SD, Freije JM and López-Otín C: Matrix metalloproteinase Mmp-1a is dispensable for normal growth and fertility in mice and promotes lung cancer progression by modulating inflammatory responses. J Biol Chem 288: 14647-14656, 2013.

30. Yang B, Gao J, Rao Z, Zhang B, Ouyang W and Yang C: Antisense oligonucleotide targeting matrix metalloproteinase-7 (MMP-7) changes the ultrastructure of human A549 lung adenocarcinoma cells. Ultrastruct Pathol 35: 256-259, 2011.

31. Bartling B, Rehbein G, Schmitt WD, Hofmann H-S, Silber R-E and Simm A: S100A2-S100P expression profile and diagnosis of non-small cell lung carcinoma: impairment by advanced tumour stages and neoadjuvant chemotherapy. Eur J Cancer 43: 1935-1943, 2007.

32. Hung WC, Tseng WL, Shiea J and Chang HC: Skp2 overexpression increases the expression of MMP-2 and MMP-9 and invasion of lung cancer cells. Cancer Lett 288: 156-161, 2010.

33. Sauter W, Rosenberger A, Beckmann L, Kropp S, Mittelstrass K, Timofeeva M, Wölke G, Steinwachs A, Scheiner D, Meese E, et al: Matrix metalloproteinase 1 (MMP1) is associated with early-onset lung cancer. Cancer Epidemiol Biomarkers Prev 17: 1127-1135, 2008.

34. Soon WW, Miller LD, Black MA, Dalmasso C, Chan XB, Pang B, Ong CW, Salto $\square$ Tellez M, Desai KV and Liu ET: Combined genomic and phenotype screening reveals secretory factor SPINK1 as an invasion and survival factor associated with patient prognosis in breast cancer. EMBO Mol Med 3: 451-464, 2011.

35. Sunaga N, Kohno T, Yanagitani N, Sugimura H, Kunitoh H, Tamura T, Takei Y, Tsuchiya S, Saito R and Yokota J: Contribution of the NQO1 and GSTT1 polymorphisms to lung adenocarcinoma susceptibility. Cancer Epidemiol Biomarkers Prev 11: 730-738, 2002.

36. Chao C, Zhang ZF, Berthiller J, Boffetta P and Hashibe M: NAD(P) H:quinone oxidoreductase 1 (NQO1) Pro187Ser polymorphism and the risk of lung, bladder, and colorectal cancers: A meta-analysis. Cancer Epidemiol Biomarkers Prev 15: 979-987, 2006

37. Du M, Wang G, Barraclough R and Rudland P: P0017 S100P regulates cytoskeleton dynamics to promote cell migration and metastasis. Eur J Cancer 51: e6-e7, 2015.

38. Whiteman HJ, Weeks ME, Dowen SE, Barry S, Timms JF, Lemoine NR and Crnogorac-Jurcevic T: The role of S100P in the invasion of pancreatic cancer cells is mediated through cytoskeletal changes and regulation of cathepsin D. Cancer Res 67: 8633-8642, 2007. 
39. Agarwal R, D'Souza T and Morin PJ: Claudin-3 and claudin-4 expression in ovarian epithelial cells enhances invasion and is associated with increased matrix metalloproteinase-2 activity. Cancer Res 65: 7378-7385, 2005.

40. Ateeq B, Tomlins SA, Laxman B, Asangani IA, Cao Q, Cao X, Li Y, Wang X, Feng FY, Pienta KJ, et al: Therapeutic targeting of SPINK1-positive prostate cancer. Sci Transl Med 3: 72ra17, 2011.

41. Lee CG, Ren J, Cheong IS, Ban KH, Ooi LL, Yong Tan S, Kan A, Nuchprayoon I, Jin R, Lee KH, et al: Expression of the FAT10 gene is highly upregulated in hepatocellular carcinoma and other gastrointestinal and gynecological cancers. Oncogene 22: 2592-2603, 2003.

42. Yan DW, Li DW, Yang YX, Xia J, Wang XL, Zhou CZ, Fan JW, Wen YG, Sun HC, Wang Q, et al: Ubiquitin D is correlated with colon cancer progression and predicts recurrence for stage II-III disease after curative surgery. Br J Cancer 103: 961-969, 2010.

43. Kim H, Watkinson J, Varadan V and Anastassiou D: Multi-cancer computational analysis reveals invasion-associated variant of desmoplastic reaction involving INHBA, THBS2 and COL11A1 BMC Med Genomics 3: 51, 2010.

44. Li J, Tu Y, Jiang L, Xu H and Zhang S: Expression and significance of Snail and Claudin-3 in non-small cell lung cancer. Zhongguo Fei Ai Za Zhi 15: 583-590, 2012 (In Chinese).
45. Yang L, Chen Y, Cui T, Knösel T, Zhang Q, Albring KF, Huber O and Petersen I: Desmoplakin acts as a tumor suppressor by inhibition of the Wnt/ $\beta$-catenin signaling pathway in human lung cancer. Carcinogenesis 33: 1863-1870, 2012.

46. Lin CY, Tsai PH, Kandaswami CC, Lee PP, Huang CJ, Hwang JJ and Lee MT: Matrix metalloproteinase-9 cooperates with transcription factor Snail to induce epithelial-mesenchymal transition. Cancer Sci 102: 815-827, 2011.

47. Nerlov C: The C/EBP family of transcription factors: A paradigm for interaction between gene expression and proliferation control. Trends Cell Biol 17: 318-324, 2007.

48. Armstrong DA, Phelps LN and Vincenti MP: CCAAT enhancer binding protein-beta regulates matrix metalloproteinase-1 expression in interleukin-1beta-stimulated A549 lung carcinoma cells. Mol Cancer Res 7: 1517-1524, 2009.

This work is licensed under a Creative Commons Attribution-NonCommercial-NoDerivatives 4.0 International (CC BY-NC-ND 4.0) License. 